INRA Prod. Anim., 1999, $12(2), 135-146$
P. CHEMINEAU, G. BARI L,

B. LEBOEUF*, M.C. MAUREL, F. ROY, M. PELLICER-RUBIO, B. MALPAUX, Y. COGNIE

INRA Stati on de Physi ologi e de la Reproduction des Mammi fères Domesti ques, URA CNRS 1291, 37380 Nouzilly

*INRA Stati on Expéri mentale d'I nsémi nati on Arti fici elle 86480 Roui llé

Phi li ppeChemi neau@tours.inra.fr

\section{Implications \\ des progrès récents en physiologie de la reproduction pour la conduite de la reproduction dans l'espèce
caprine dans l'espe
caprine}

II existe plusieurs voies pour mieux maîtriser la reproduction dans l'espèce caprine : traitements lumineux pour supprimer le caractère saisonnier de la reproduction, traitements de synchronisation des chèvres, techniques de conservation de la semence et de production d'embryons. Cet article fait le point, pour chacune d'elles, sur les progrès récents les plus significatifs.

\begin{abstract}
Résumé
Le contrôle de la reproduction caprine est intéressant pour des raisons techniques (synchronisation des mise bas, ajustements aux disponibilités fourragères ou aux contraintes économiques), et pour des raisons génétiques (identification et dissémination des génotypes améliorateurs). L'utilisation de rythmes photopériodiques accélérés conduit à une augmentation très importante des doses produites pour l'insémination artificielle (IA) et évite l'apparition d'une contre-saison sexuelle. L'identification récente d'une lipase bulbo-uréthrale dans le sperme de bouc offre de nouvelles perspectives pour la conservation de la semence. Chez la chèvre, les traitements " Iumière + jours courts " permettent également l'induction de cycles oestriens et ovulatoires à contre-saison, ce qui conduit à une amélioration de la fertilité des troupeaux. En revanche, I'utilisation répétée de eCG (autrefois appelée PMSG) provoque l'apparition d'anticorps, retarde le moment de l'ovulation et induit une baisse de fertilité après IA, lorsqu'elle est réalisée après un délai fixe après la fin du traitement. Toutes les étapes de la production d'embryons, de leur congélation et de leur transfert sont maintenant maîtrisées et permettent d'atteindre des taux satisfaisants de chevreaux nés par femelle donneuse, qui sont compatibles avec le développement de la technique dans le but d'échanger du matériel génétique entre les pays. La production in vitro d'embryons permet des taux élevés de développement après maturation et fécondation in vitro des ovocytes et pourrait permettre l'obtention de populations synchrones de zygotes au stade unicellulaire, nécessaire à l'application des biotechnologies.
\end{abstract}

Comme chez les autres espèces d'animaux domestiques, le contrôle de la reproduction chez les caprins offre des avantages pour les exploitations agricoles mais aussi à l'échellede la population dans laquelle un progrès génétique est possible. Le premier avantage est le choix d'une période de mise bas à une période précise de l'année (pour s'ajuster à des conditions favorables de l'environnement extérieur, par exemple à la saison de pousse de l'herbe, ou à une meilleure période pour la commercialisation des produits). Le deuxième avantage réside dans la synchronisation des mise bas sur une période de temps limité, ce qui facilite la surveillance des naissances et conduit à une réduction de la mortalité des chevreaux, à la constitution de groupes homogènes de mères et de jeunes permettant de les alimenter en lots, et a une optimisation de la main d'oeuvre nécessaire aux soins apportés aux animaux. Le troisième avantage du contrôle de la reproduction caprine est qu'elle permet la manipulation et le stockage du matériel génétique. L'insémination artificielle (IA), même utilisée sur une petite échel- 
le, permet l'établissement de connexions entre les troupeaux, ce qui accroît fortement l'efficacité de l'indexation des mâles. Une estimation précoce et précise de la valeur génétique des jeunes boucs est ainsi possible. Une fois identifiés, les mâles améliorateurs peuvent être utilisés dans un grand nombre de troupeaux. Le transfert d'embryons accroît le nombre de descendants d'une femelle génétiquement supérieure et constitue une méthode efficace et sûre pour échanger du matériel génétique sans propager de maladies. Enfin, la production in vi tro d'embryons permettra, sans doute dans un avenir assez proche, d'avoir accès au génome d'embryons au stade unicellulaire.

Dans cet article, nous avons délibérément choisi de présenter un nombre limité de résultats pour des techniques qui ont connu des progrès significatifs ces dernières années. Ces techniques sont utilisables dans des systèmes d'élevage intensifs pour lesquels le revenu par chèvre est élevé, en général en raison du prix élevé de vente du lait.

\section{Production de sperme et technologie de la semence}

L'utilisation, chez les boucs, de traitements photopériodiques permet de supprimer le probleme du saisonnement de la production spermatique. Développés initialement chez le bélier, les rythmes photopériodiques accélérés (alternances entre un ou deux mois de

Figure 1. Poids testiculaires moyens de boucs Alpins et Saanen, traités ( $n=6)$ ou non ( $n=6$ ) avec un rythme lumineux accéléré d'une période de 4 mois (d'après $B$. Leboeuf et $P$. Chemineau, résultats non publiés).

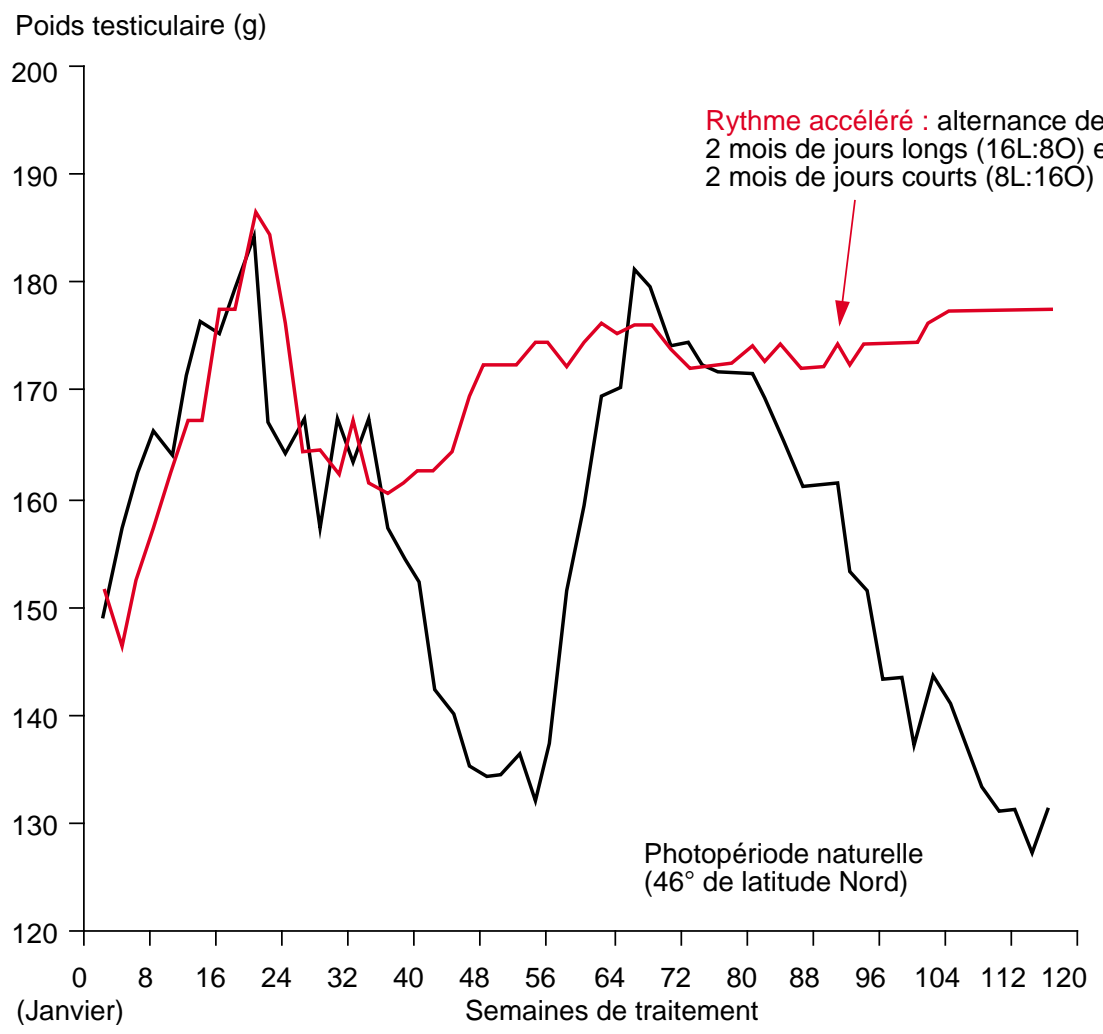

jours longs ( $16 \mathrm{~h}$ lumière : $8 \mathrm{~h}$ obscurité) et un ou deux mois de jours courts (8h lumière : $16 \mathrm{~h}$ obscurité)) abolissent les variations saisonnières de poids testiculaire et de production spermatique. Des boucs Alpins et Saanen, soumis pendant trois années consécutives à de tels traitements photopériodiques, présentent une amélioration considérable de tous les paramètres de production spermatique, en comparaison avec des boucs témoins maintenus sous photopériode naturelle (Delgadillo et al 1993). Avec une collecte bihebdomadaire, le nombre total de spermatozoïdes collectés est augmenté de $61 \%$ (Delgadillo et al 1991). La semence ne présente plus les variations saisonnières importantes de qualité observées chez les boucs témoins. Le nombre total de doses pour I'IA produites pendant les deux premières années de traitement est beaucoup plus élevé ( $+62 \%)$ que celui produit par les boucs témoins. La fécondité de la semence n'est pas significativement modifiée par le traitement, en dépit d'une petite baisse de fertilité dans l'un des groupes de boucs traités (Delgadillo et al 1992).

A la suite de ces résultats, il est apparu que le rythme de collecte (bihebdomadaire) pouvait être accru chez les mâles traités. II a donc été décidé de comparer la production spermatique des boucs traités, collectés quatre fois par semaine toute l'année, à la production spermatique des boucs témoins, collectés quatre fois par semaine, seulement de septembre à fevrier, comme dans la conduite habituelle des animaux. Au cours des 24 mois du traitement, comme attendu, le poids testiculaire des boucs est resté constant, au niveau maximum de la pleine saison sexuelle, tandis que le poids testiculaire des mâles témoins suivait les variations saisonnières normales, comme observé auparavant (figure 1).

De ce fait, la production spermatique, mesurée par le nombre total de spermatozoïdes produits, ou par le nombre de doses d'IA, a été significativement accru par le traitement ( 1106 vs 1556 doses par bouc et par an). La fécondité de ces doses d'IA était plus faible, bien que non significativement, que celle des doses produites par les boucs témoins (tableau 1).

Un tel accroissement de la production spermatique a probablement pour origine des changements inattendus dans les processus spermatogenétiques. Les boucs subissant ces traitements photopériodiques présentent une augmentation significative du nombre de leurs spermatogonies (les cellules souches à l'origine de la lignée germinale) alors que les divisions spermatogenétiques sont maintenues aux rendements maximaux observés pendant la saison sexuelle (Delgadillo et al 1995). En permettant la collecte de la semence tout au long de l'année, au lieu des six mois habituels chez les boucs maintenus en photopériode naturelle, ces traitements permettent d'accélérer la vitesse de constitution du stock de doses d'IA produites par les jeunes boucs au cours des 2,5 ans du testage sur descendance. Ce traitement photopériodique est désormais utilisé pour accroître la production spermatique des boucs du schéma national d'amélioration génétique. 
Les résultats les plus récents dans le domaine de la technologie de la semence ont trait à l'identification d'une enzyme du plasma séminal qui diminue la survie in vi tro des spermatozoîdes. Le jaune d'oeuf ou le lait écrémé sont fréquemment utilisés dans les dilueurs pour la semence mammalienne du fait de leur rôle protecteur contre le " choc au froid " des spermatozoïdes. Toutefois, la cryopréservation du sperme de bouc dans de tels milieux impose que la quasi totalité du plasma séminal soit éliminé avant la dilution du sperme (méthode dite du " lavage "), afin d'accroître les taux de survie des spermatozoïdes après décongélation. La sécrétion des glandes bulbo-uréthrales (BUS) est la fraction du plasma séminal du bouc responsable de la détérioration de la viabilité des spermatozoïdes dans le jaune d'oeuf (Roy 1957) et dans les dilueurs à base de lait (Nunes et al 1982). C'est actuellement un dilueur à base de lait qui est utilisé dans la pratique (Leboeuf et al 1998). L'enzyme coagulant le jaune d'oeuf (EYCE) provenant des BUS possède une activité phospholipase $A$ et peut hydrolyser les lécithines du jaune d'oeuf en acides gras et Iysolécithines (Roy 1957, Iritani et Nishikawa 1972) qui sont toxiques pour les spermatozoïdes (Aamdal et al 1965). Dans le cas de I'utilisation du lait écrémé comme dilueur de la semence, le composé des BUS responsable de cet effet vient d'être purifié et identifié comme étant une glycoprotéine de 55 à 60 kDa (baptisée BUSgp60) qui possède une activité triglycéride lipase (Pellicer-Rubio et al 1997). Cette BUSgp60 provoque une diminution du pourcentage de spermatozoïdes mobiles, une détérioration de la qualité du mouvement spermatique, une rupture de l'acrosome et une mort cellulaire des spermatozoïdes épidydimaires de bouc, dilués dans
Figure 2. Effets dose-dépendant comparés du BUSgp60 purifié (symboles rouges) et du BUS (symboles noirs) sur les paramètres de qualité de spermatozoïdes épidydimaires de boucs, dilués dans du lait écrémé, après 60 minutes d'incubation à $37^{\circ} \mathrm{C}$ Les doses de BUS sont exprimées en doses équivalentes de BUSgp60 (d'après Pellicer-Rubio et al 1997).
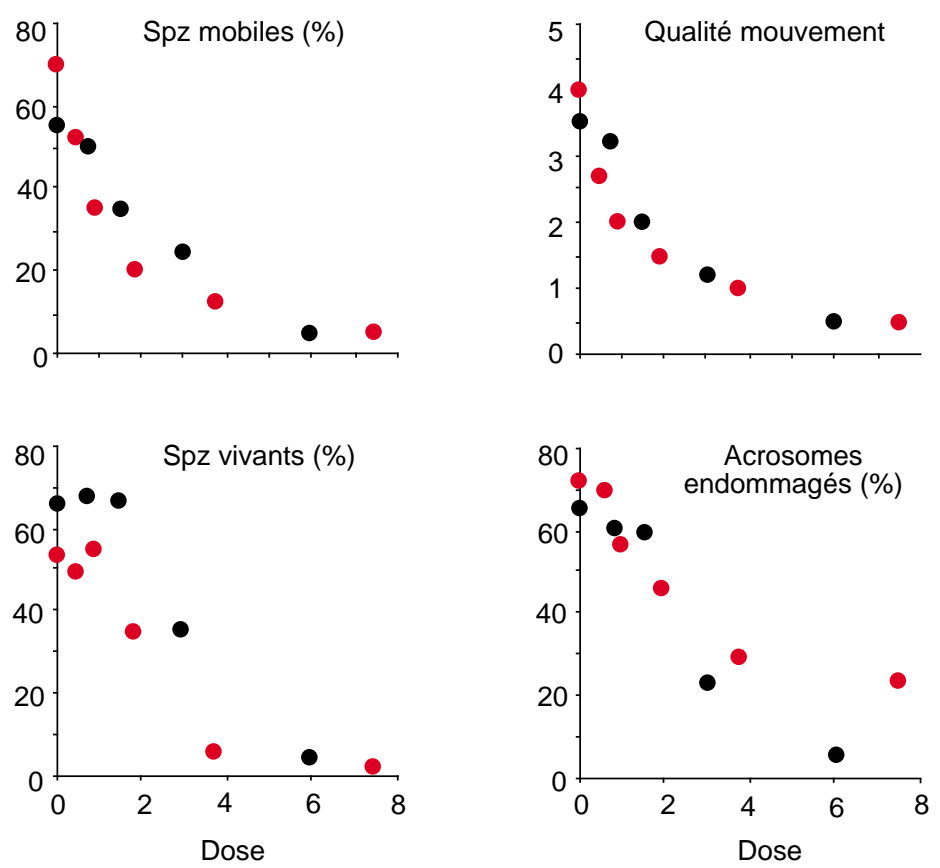

du lait écrémé (figure 2 ; Pellicer-Rubio et al 1997, Pellicer-Rubio et Combarnous 1998). Ces résultats ouvrent la possibilité d'inhiber spécifiquement la lipase BUSgp60 dans des dilueurs à base de lait, afin d'éviter l'étape du "lavage " de la semence, assez traumatisante pour les spermatozoïdes, actuellement utilisée avant la congélation. De plus, I'utilisation

Tableau 1. Production de semence et fertilité après IA chez des boucs témoins (soumis à l'éclairement naturel), ou traités avec des alternances photopériodiques rapides de jours longs (JL, 16 heures d'éclairement par jour) et de jours courts (JC, 8 heures d'éclairement par jour). Les IA sont effectuées dans chaque troupeau après répartition des femelles à inséminer dans chacun des groupes de mâles témoins ou traités (d'après Delgadillo et al 1992 et B. Leboeuf et $P$. Chemineau, résultats non publiés).

\begin{tabular}{|c|c|c|c|c|}
\hline & \multicolumn{4}{|c|}{ Groupes expérimentaux } \\
\hline $\begin{array}{l}\text { Expérience } 1 \text { (Delgadillo et al 1992) } \\
\text { - Nombre de boucs } \\
\text { - Rythme de collecte } \\
\text { - Nombre de doses d'IA (à } 200 \times 10^{6} \mathrm{spz} / \text { dose) } \\
\text { produites par bouc et par an } \\
\text { - Fertilité ( } \% \text { mise bas) } \\
\text { ( } 1599 \text { chèvres dans } 58 \text { troupeaux) }\end{array}$ & $\begin{array}{c}\text { Témoin } \\
\text { (éclairement naturel) } \\
6 \\
2 \text { éjaculats/ semaine } \\
253 \\
62,5\end{array}$ & 2 éjacu & aine & $\begin{array}{c}2 \text { mois J L } \\
2 \text { mois J C } \\
6 \\
2 \text { éjaculats/ semaine } \\
391 \\
57,8\end{array}$ \\
\hline $\begin{array}{l}\text { Expérience } 2 \text { (B. Leboeuf et } P \text {. Chemineau, nor } \\
\text { - Nombre de boucs } \\
\text { - Rythme de collecte } \\
\text { - Nombre de doses d'IA (à } 100 \times 10^{6} \mathrm{spz} / \text { dose) } \\
\text { produites par bouc et par an } \\
\text { - Fertilité (\% mise bas) } \\
\text { ( } 785 \text { chèvres dans } 25 \text { troupeaux) }\end{array}$ & $\begin{array}{l}4 \text { éjacu } \\
\text { de Se }\end{array}$ & $\begin{array}{l}\text { semaine } \\
\text { évrier }\end{array}$ & & $\begin{array}{l}6 \\
\text { llats/ semaine } \\
\text { ite l'année }\end{array}$ \\
\hline
\end{tabular}


d'inhibiteurs spécifiques de la BUSgp60 pourrait améliorer la cryopréservation de la semence de bouc non lavée.

\section{Induction d'une cyclicité en dehors de la saison sexuelle chez la chèvre par des traite- ments photopériodiques}

Des traitements appropriés de mélatonine peuvent être utilisés afin que les animaux percoivent des jours courts alors que leurs yeux perçoivent les jours longs du printemps ou de l'été (Chemineau et al 1992, Deveson et al 1992a, Malpaux et al 1993, Von BrackelBodenhausen et al 1994), dans le but d'avancer les activités ovulatoire et de comportement d'oestrus. Cependant, Iorsqu'il est appliqué seul chez les races à fort saisonnement, le traitement par la mélatonine ne permet d'avancer la saison sexuelle que de 1,5 mois. Ce résultat n'est pas satisfaisant, en particulier en France où beaucoup d'éleveurs souhaitent induire une saison sexuelle complète à contre-saison (d'avril à juillet). Pour y par- venir, le traitement mélatonine doit être précédé par au moins deux mois d'un traitement quotidien composé de jours longs réels (Deveson et al 1992a), ou de deux périodes d'éclairement supplémentaires (figure 3 ; Chemineau et al 1992). De tels " jours longs " ("J L ") procurent sans doute le signal photoperiodique du début de la saison sexuelle annuelle et aussi rétablissent la sensibilité à la mélatonine (Chemineau et al 1992, Malpaux et al 1993). Chez les chèvres Alpines laitières maintenues en chèvrerie ouverte, l'utilisation de la succession " J L " + mélatonine, suivie par un " effet bouc " avec des mâles ayant subi le même traitement lumineux, permet l'induction d'activités ovulatoire et de comportement d'oestrus suffisantes pour atteindre une fertilité et une prolificité voisine de celles observées pour une saison sexuelle annuelle normale (Chemineau et al 1996).

Le traitement " JL " doit durer au moins deux mois, la concentration de mélatonine fournie par le traitement doit être optimale et, pour la lutte naturelle, le moment d'introduction des boucs traités avec "J L " + mélatonine doit être situé de 35 à 70 jours après la fin du traitement lumineux (Chemineau et al 1996).

Figure 3. Traitements " photopériodiques " pouvant être appliqués en chèvrerie ouverte, qui utilisent la succession de " jours longs " et de " jours courts ", suivis d'un effet bouc. Le traitement (a), avec retour à la photopériode naturelle seule, peut être appliqué tôt dans l'année et le traitement (b), qui utilise la mélatonine à la fin de la période de "jours longs ", peut être appliqué plus tard dans l'année.

en contre-saison,

un traitement

lumineux permet

d'induire la

cyclicité. S'il est

appliqué tôt en

saison,

l'administration

de mélatonine

n'est pas

nécessaire
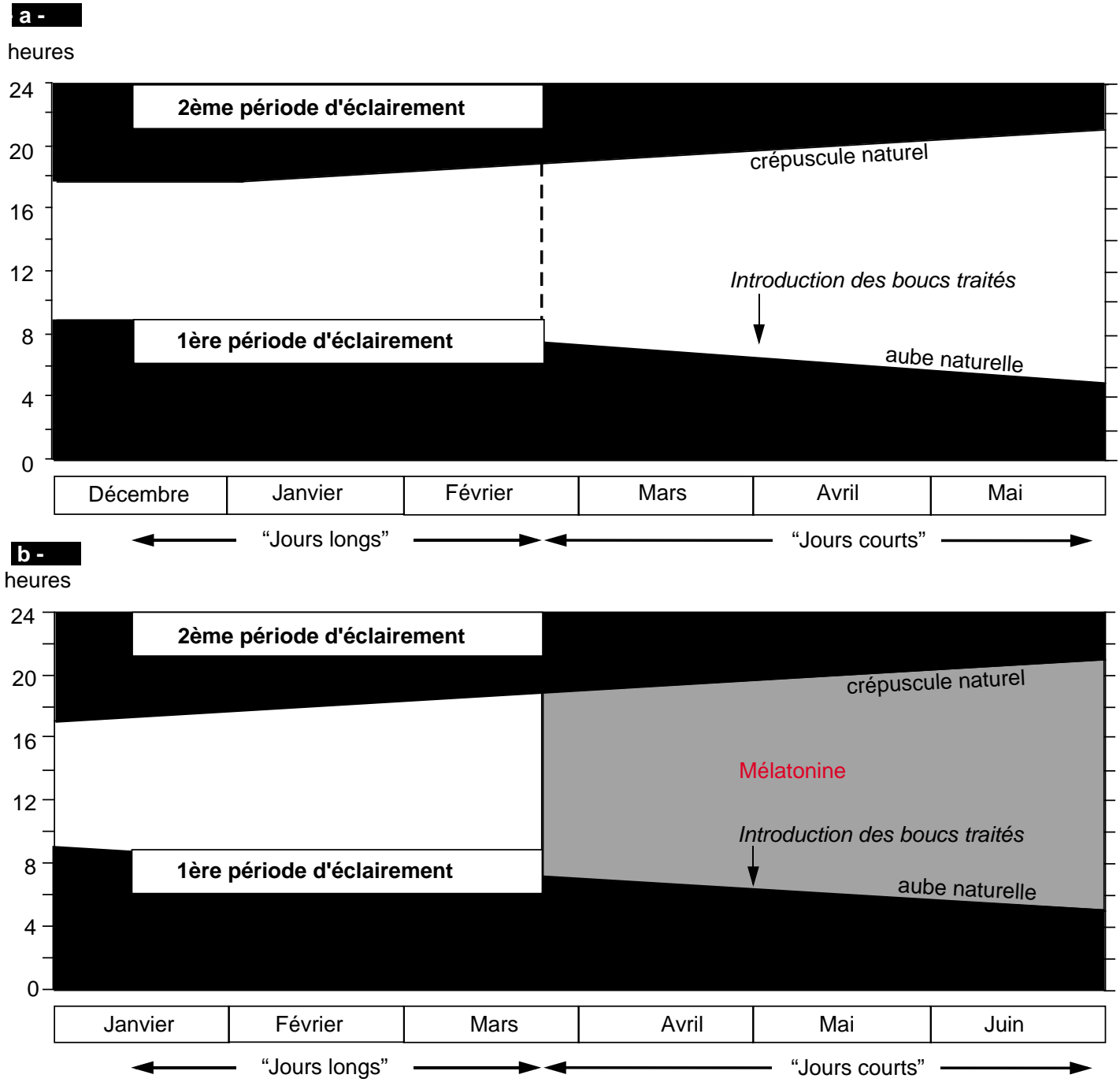

INRA Productions Ani males, mai 1999 
Tableau 2. Fertilité et taille de portée de chèvres après traitement photopériodique seul dans plusieurs troupeaux et comparaison intratroupeau du traitement avec et sans mélatonine.

\begin{tabular}{|c|c|c|c|}
\hline & $\begin{array}{l}\text { Nombre de femelles } \\
\text { (mise bas) }\end{array}$ & Fertilité & $\begin{array}{c}\text { Taille de portée } \\
\text { (chevreaux nés/ mise bas) }\end{array}$ \\
\hline $\begin{array}{l}\text { Traitement phopériodique seul } \\
\text { Lutte naturelle, } 20 \text { troupeaux } \\
\text { (Groupe Reproduction Caprine 1996) }\end{array}$ & 3236 & $76,8 \%$ & 1,83 \\
\hline $\begin{array}{l}\text { Traitement photopériodique avec et sans mélatonine } \\
\text { Lutte naturelle } 1996+1997,1 \text { seul troupeau } \\
\text { (B. Leboeuf et P. Chemineau, non publié) } \\
\text { Avec mélatonine } \\
\text { Sans mélatonine }\end{array}$ & $\begin{array}{l}126 \\
115\end{array}$ & $\begin{array}{l}75,3 \% \\
73,0 \%\end{array}$ & $\begin{array}{l}2,07 \\
2,00\end{array}$ \\
\hline
\end{tabular}

Si ces conditions sont respectées, le pic des fécondations se situe environ dix jours après l'introduction des boucs et quelques femelles sont fécondées sur retour, un cycle plus tard. Ces traitements photopériodiques peuvent modifier la vitesse de pousse du pelage (Gebbie 1993), et l'éclairement des chèvres pendant la gestation (ce qui n'a pas lieu en conduite normale) est susceptible de retarder d'environ 4 mois la puberté des chevrettes nées des chèvres recevant l'éclairement (Deveson et al 1992b) ; il n'est donc pas recommandé.

Plus récemment, il a été montré que lorsque le traitement lumineux est appliqué très tôt en saison (fin aux alentours de la fin mars), le traitement mélatonine n'est pas nécessaire et le retour à l'éclairement naturel après les "J L " est suffisant pour obtenir des taux de fertilité satisfaisants (tableau 2).

\section{Synchronisation hormonale de l'oestrus}

Les traitements hormonaux des chèvres afin d'induire un début synchrone des comportements d'oestrus et des ovulations sur une durée limitée dans le temps après la fin du traitement constituent des pré-requis pour l'utilisation de l'insémination artificielle (IA). L'association entre un progestatif (délivré par une éponge vaginale ou un implant sous-cutané, Bretzlaff et Madrid 1985), un analogue de prostaglandine et la PMSG (maintenant appelée eCG pour "equine Chorionic Gonadtotrophin "), reste le moyen le plus efficace pour atteindre ces objectifs. Ces traitements sont maintenant largement utilisés dans le monde entier pour contrôler la reproduction des chèvres. Leur utilisation pour réaliser une IA à l'aveugle, c'est-à-dire sans détection des chaleurs, a été testée sur des milliers de chèvres et permis d'obtenir des taux de fertilité élevés (Leboeuf et al 1998). Ce traitement peut également être appliqué aux chevrettes si certaines conditions, notamment de développement corporel, sont respectées (Corteel et al 1993).

Des expériences récentes ont été conduites afin d'essayer de réduire la variabilité de l'intervalle retrait de l'éponge- début de l'oestrus. $\mathrm{Ni}$ l'augmentation de la quantité d'acétate de fluorogestone (FGA) délivrée par l'éponge, ni l'utilisation d'implants sous-cutanés ne réduisent cette variabilité ( $F$ reitas et al 1996a et 1997a). Pas plus le nombre de corps jaunes que le nombre et la taille des follicules présents sur l'ovaire avant et/ou pendant le traitement FGA n'influencent la réponse (Freitas et al 1996b). Enfin, il a été observé que, lors de cycles naturels, la variabilité de l'intervalle

Figure 4. Relations entre l'intervalle retrait de l'éponge -début de l'oestrus et le nombre de traitements reçus par les chèvres, et entre cet intervalle et la fertilité ( $m \pm$ esm, d'après Baril et al 1993).

Intervalle retrait de l'éponge - oestrus (h)

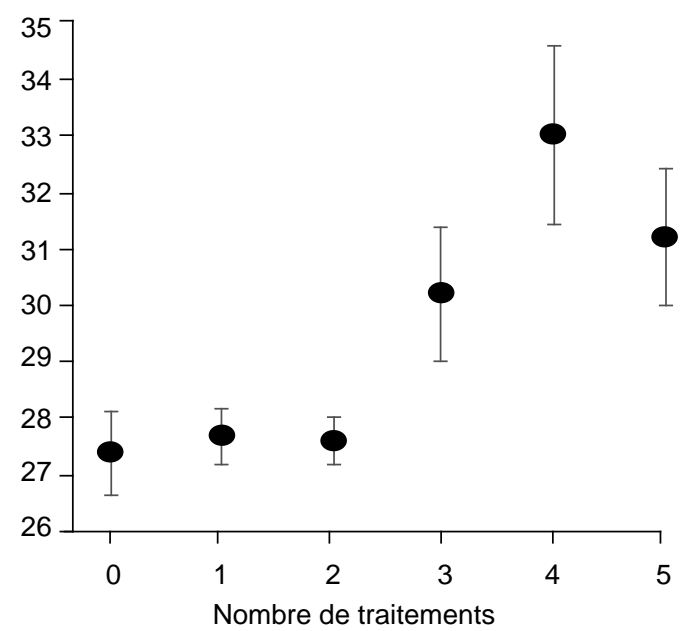

Fertilité (\% mise bas)

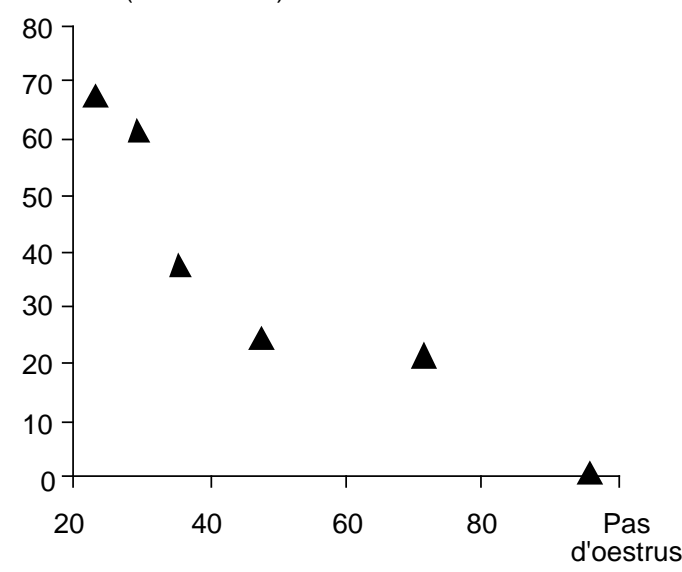

Intervalle retrait de l'éponge - oestrus (h) 
fin de lutéolyse -début d'oestrus ou -début du pic préovulatoire de LH était supérieure à celle observée après un traitement FGA-analogue de prostaglandines (Freitas et al 1997b). Ainsi, des améliorations du traitement " classique " seront vraisemblablement difficiles à obtenir.

Paradoxalement, quand eCG est utilisée de facon répétée sur les mêmes femelles, son efficacite diminue.

Dans un troupeau Saanen de 169 chèvres pour lequel la période de reproduction annuelle est souhaitée en contre-saison après un traitement FGA-eCG, la proportion de chèvres qui viennent en oestrus et leur fertilité sont inférieures chez les multipares que chez les primipares ( 64 vs $99 \%$ et 34 vs $67 \%$, respectivement). Quand les chèvres sont traitées pour la seconde fois dans la même année, la proportion d'entre elles venant en oestrus est plus faible qu'après le premier traitement ( 45 vs $71 \%$, Baril et al 1992). Cette situation est due à l'apparition d'anticorps anti-eCG (Roy et al 1995 et 1998 ; voir ci-après). Des résultats aussi drastiques dans un seul troupeau ont incité à effectuer deux larges essais dans plusieurs troupeaux privés, utilisant l'association FGA-eCG, avec une IA "classique "avec de la semence congelée. Dans le premier essai, un comportement d'oestrus est induit chez la quasi totalité des chèvres traitées $(98,1 \%$ des 368 Alpines et 272 Saanen de 19 troupeaux privés) de 24 à 72 heures après le retrait de l'éponge. L'apparition du début de l'oestrus après retrait est affecté par le nombre de traitements reçus au préalable par la femelle et semble s'accroître de façon très importante après le second traitement (figure 4). La fertilité, mais pas la prolificité, après IA est négativement corrélée à l'intervalle retrait d'éponge -début d'oestrus $(R=0,92)$. La fertilité des chèvres qui sont venues en oestrus au-delà de 30 heures après le retrait de l'éponge est significativement plus faible que celle des chèvres dont l'oestrus est apparu entre 24 et 30 heures post-retrait (33 vs $65 \%$ respectivement, figure 4, Baril et al 1993). Ce retard dans l'apparition de l'oestrus est associé à un retard dans l'apparition du pic préovulatoire de LH (Maurel et al 1992) et à un retard du moment d'ovulation (Leboeuf et al 1993 et 1996). Dans le second essai, la liaison eCG (mesurée chez 524 chèvres laitières de 17 troupeaux privés) avant le début du traitement est significativement plus faible chez les chèvres des troupeaux dans lesquels les traitements n'avaient jamais été utilisés que dans le reste de l'échantillon et ne dépend pas de l'âge des femelles. La liaison est superieure chez les femelles ayant reçu auparavant de 2 à 5 traitements, comparée à celle des femelles ayant reçu un seul traitement ou pas du tout ( 3 vs $10 \%$ ). Sur le plan individuel, la proportion de chèvres manifestant un début d'oestrus plus de 30 heures après retrait est plus élevé ( 38 vs $7 \%$ ) et la fertilité est plus faible (51 vs $66 \%$ sur

Figure 5. Evolution de la réponse immunitaire anti-eCG chez huit chèvres Alpines qui reçoivent $500 \mathrm{UI}$ de eCG à JO. Les huit chèvres sont considérées comme représentatives de tout le groupe étudié. La concentration d'anticorps anti-eCG est déterminée par dosage ELISA. Chaque valeur de concentration a été déterminée en double (d'après Roy et al 1995).

Concentration d'anticorps anti eCG ( $\mu \mathrm{g} / \mathrm{ml})$

100

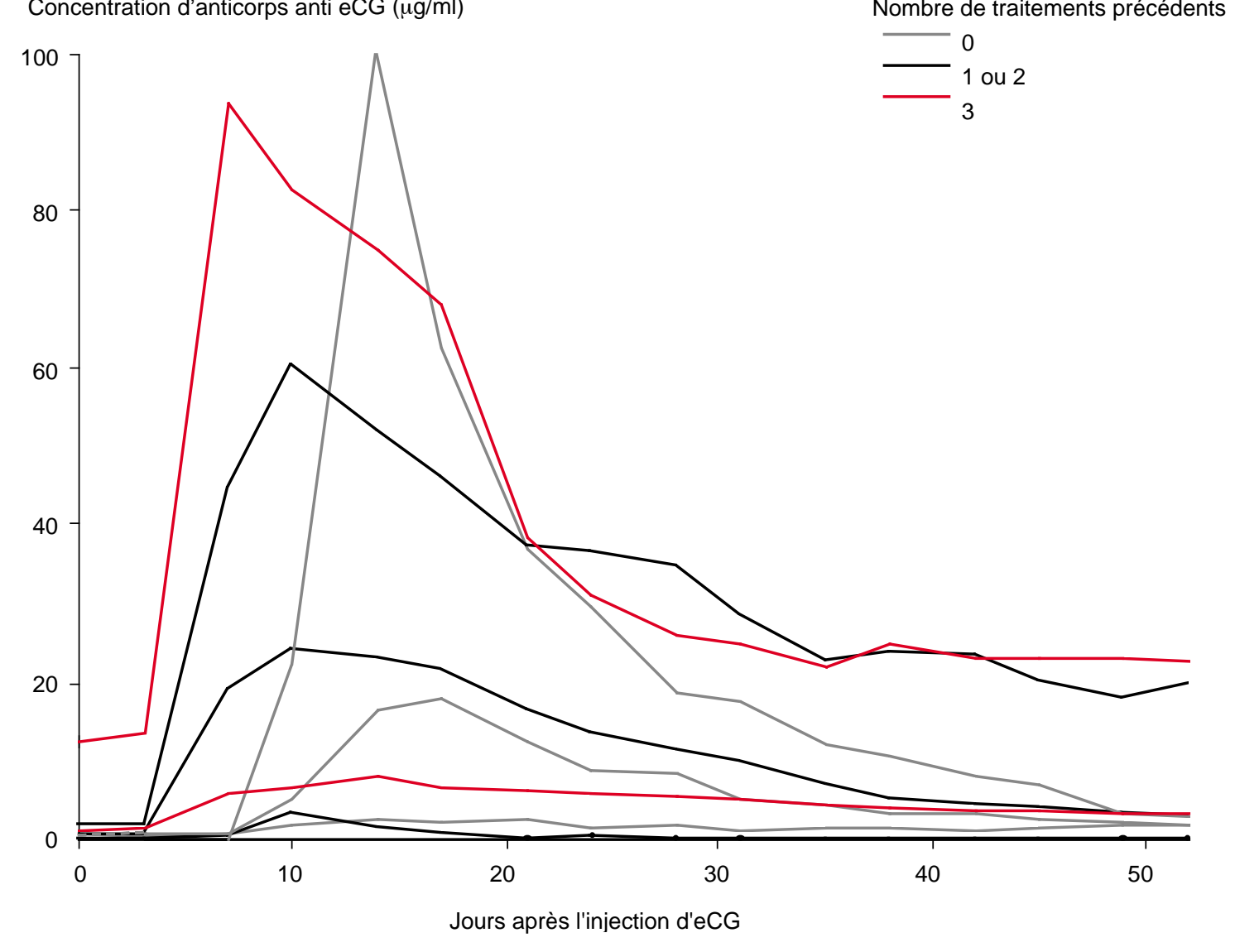

INRA Productions Animales, mai 1999 
166 vs 353 femelles) quand la liaison eCG est plus élevée (plus de $10 \%$ de liaison de eCG radioactive vs moins de $5 \%$ ). Vingt-cinq jours après l'injection de eCG, le taux de liaison de eCG est accru (7 \% avant injection vs $28 \%$ après injection) et corrélé avec le taux de liaison mesuré avant traitement (Baril et al 1996b).

Des études complémentaires ont été conduites pour évaluer l'induction d'une réponse immunitaire humorale anti-eCG après une injection de $500 \mathrm{UI}$ d'eCG. Quinze chèvres ont été traitées pour la première fois avec de l'eCG et montrent une concentration élevée d'anticorps dix jours après l'injection. Des chèvres $(n=29)$ ayant déià été traitées auparavant (une ou plusieurs fois) avec eCG montrent une cinétique identique de leur réponse immunitaire humorale, sauf qu'elles manifestent une augmentation plus précoce de leur concentration d'anticorps à $J$, ainsi qu'une phase plus longue de diminution des taux circulants (figure 5, Roy et al 1995). Que que soit le nombre de traitements reçus au préalable, les chèvres diffèrent fortement les unes des autres par leur concentration d'anticorps anti-eCG. En effet, la concentration maximale varie de 0,7 à $102 \mu \mathrm{g} / \mathrm{ml}$ chez les chèvres traitées pour la première fois, et de 3,0 à $219 \mu \mathrm{g} / \mathrm{ml}$ chez les chèvres traitées plusieurs fois. Néanmoins, en dépit de l'hétérogénéité des niveaux de sécrétion d'anticorps, les résultats montrent que la concentration moyenne d'anticorps mesurée avant le traitement s'accroît significativement $(P<0,05)$ avec le nombre de traitements eCG antérieurs. Cette variabilité est sous contrôle génétique (Roy et al 1998). Les fortes concentration résiduelles d'anticorps (résultant des traitements effectués les années antérieures) entraînent une baisse de fertilité après un nouveau traitement et IA des femelles, au contraire des concentrations mesurées pendant la réponse immunitaire (à J 10 et J 25).

La fertilité des femelles venant en oestrus plus de 30 heures après retrait de l'éponge (qui représentent $18 \%$ de l'échantillon dans les expériences antérieures) est faible sans doute à cause de leur ovulation retardée et parce que la semence congelée a une durée de vie limitée. Quand ces femelles sont inséminées artificiellement, plus tard, de façon adéquate avec le moment de détection de l'oestrus, leur fertilité n'est pas altérée (B. Leboeuf et $G$. Baril, résultats non publiés). Aussi, I'IA des seules femelles qui ont été détectées en oestrus à 30 heures après retrait de l'éponge est recommandée.

Par ailleurs, que ce soit pour une insémination artificielle ou naturelle, le traitement des mêmes femelles plus d'une seule fois par an n'est pas conseillé.

\section{Pseudogestations}

La fertilité des chèvres après IA peut être réduite par l'existence de pseudogestations au moment de l'induction de l'oestrus. Cette affection se caractérise par un maintien du corps jaune et l'accumulation de liquide utérin, visible à l'échographie, qui provoque une apparence de gestation. Plusieurs enquêtes utilisant l'échographie ont montré que les pseudogestations apparaissaient chez 3 à $4 \%$ des chèvres, quelquefois jusqu'à $20 \%$ dans quelques troupeaux (Mialot et al 1991, Hesselink 1993, Leboeuf et al 1994). Les pseudogestations sont reliées à la race dans certaines enquêtes (Leboeuf et al 1994) mais pas dans d'autres (Mialot et al 1991), à la méthode de reproduction (3,8\% chez 1493 chèvres traitées avec FGA/eCG vs 2,5\% chez 3774 chèvres en lutte naturelle; Mialot et al 1991), au père de la femelle $(20 \%$ des 125 filles de 5 boucs vs $0 \%$ des 326 filles de 12 boucs, dans le même troupeau ; Soulière 1991), à la parité ( $1 \%$ des nullipares vs $18 \%$ des primi ou multipares ; Hesselink 1993), et à l'âge (10\% des 280 chèvres de moins de 5 ans vs $32 \%$ des 34 chèvres de plus de 6 ans ; Hesselink 1993).

Une étude récente, effectuée chez des chèvres inséminées artificiellement, a montré que plus de $50 \%$ des pseudogestations identifiées par échographie environ 45 jours après $I A$, font suite a une mortalité embryonnaire tardive. Parmi 67 chèvres qui ont été diagnostiquées pseudogestantes 40-60 jours après IA, $54 \%$ d'entre elles avaient des taux de PSPB (une hormone placentaire) détectables, démontrant la présence de tissu foetal, 30-36 jours après I'IA (Humblot et al 1995). Ainsi, au moins $50 \%$ de ces " pseudogestations " sont la conséquence d'une mortalité embryonnaire tardive, dont les raisons restent à déterminer.

Le traitement avec un analogue des prostaglandines stoppe la pseudogestation (Hesselink 1993) et rétablit la fertilité. Toutefois, après une ou deux injections d'analogue de prostaglandines $(100 \mu \mathrm{g})$, suivies 10 jours plus tard par un traitement FGA/eCG, la fertilité à la suite d'une IA n'est que de $45 \%$ $(n=286$, Leboeuf et al 1998).

\section{Production, collecte, congé- lation et transfert des embryons in vivo}

Moins développé que dans l'espèce bovine, le transfert embryonnaire caprin est désormais utilisé principalement pour l'échange de matériel génétique au travers des frontières, du fait de la tres importante diminution du risque sanitaire lorsque les protocoles internationaux définis pour la manipulation des embryons sont respectés (Wrathall 1995). Le transfert embryonnaire est aussi utilisé dans les programmes de transgenèse caprine afin de maximiser le nombre d'embryons qui doivent être micro-injectés (Gootwine et al 1997).

Les chèvres donneuses reçoivent un traitement progestatif qui se termine par des injections d'hormones gonadotropes afin de stimuler la croissance folliculaire et d'induire la superovulation. L'utilisation de FSH (en fait des extraits hypophysaires plus ou moins purs) est maintenant largement acceptée à la place de la PMSG/eCG pour espérer atteindre des taux élevés de superovulation. Si la collecte doit être répétée sur la même femelle donneuse, de la FSH ovine ou caprine (o ou
Les

"pseudogestations " seraient pour une bonne part dues à une mortalité embryonnaire tardive. 
CFSH) doit être utilisée au lieu de FSH porcine (pFSH), du fait de l'apparition d'anticorps contre la pFSH, qui limite la réponse superovulatoire des femelles (Remy et al 1991). La oFSH peut être injectée en six à huit fois, à 12 heures d'intervalle pendant les trois ou quatre derniers jours du traitement progestatif, avec une dose totale de 16 à $21 \mathrm{mg}$ (unités standard Armour) pour les Alpines et les Saanen. Cette dose doit être adaptée au génotype. Le choix de doses constantes ou décroissantes dépend de l'origine de la préparation (Baril 1995). Un ratio $\mathrm{FSH} / \mathrm{LH}$ élevé avec $60 \%$ de F SH semble adéquat (Nowshari et al 1995). En moyenne, le nombre d'ovulations induites par de tels traitements varie de 12 à 16 par chèvre, mais une forte variabilité existe entre femelles (de 0 à 40, Brebion et al 1992) et un effet saisonnier a été décrit pour certaines races (Gootwine et al 1997).

Une des limites de ces traitements de superovulation réside dans la régression précoce du corps jaune chez 10 à $35 \%$ des femelles traitées, environ 6 à 8 jours après l'oestrus. La diminution simultanée de la progestérone plasmatique conduit à une diminution très importante du taux de collecte (Borque et al 1993). Même si l'état corporel faible des animaux peut être suspecté comme étant une des raisons de cet état de fait, les principales raisons de cette lutéolyse précoce restent inconnues. L'utilisation d'un antilutéolytique ou l'injection de progestérone ont été décrites dans la litterature, avec des taux de succès variables (revue de Baril 1995).

La bonne fécondation des ovocytes des donneuses dépend de la synchronisation des ovulations et de la méthode utilisée pour inséminer les femelles. Une réduction de la variabilité de la durée des ovulations (le temps séparant la première de la dernière ovulation)

Figure 6. Nombre de produits par femelle donneuse à chaque étape in vivo de la production, collecte et transfert d'embryons frais ou congelés (d'après Baril 1995).

Nombre par chèvre donneuse

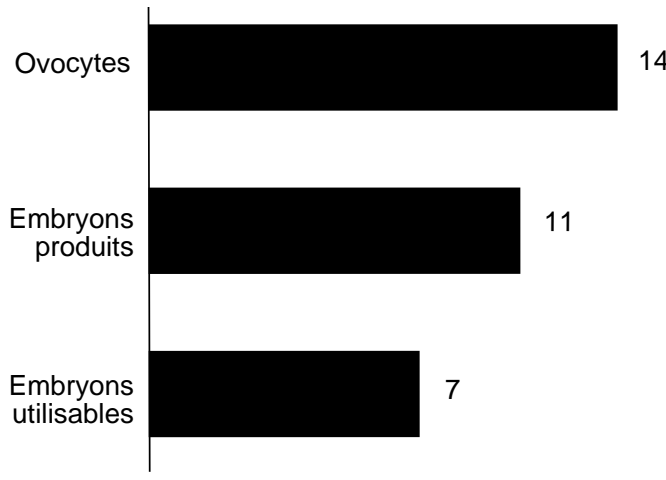

Chevreaux nés

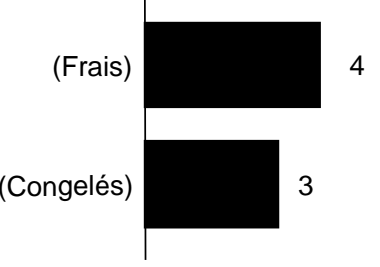

et une augmentation du taux d'ovulation ont été obtenues grâce à des injections de $\mathrm{GnRH}$ à des moments fixes après la fin du traitement progestatif (Akinlosotu et Wilder 1993, Krisher et al 1994). Une autre solution réside dans l'utilisation d'un antagoniste de $\mathrm{GnRH}$, 12 heures après le retrait des éponges, suivie d'une injection intraveineuse de $3 \mathrm{mg}$ de $\mathrm{pLH}$ 24 heures plus tard, qui mime le pic préovulatoire et permet I'IA des femelles seulement une fois, 16 heures après l'injection de LH (Baril et al 1996a). L'insémination naturelle (la saillie) peut être utilisée avec de bons résultats (fertilité voisine de $80 \%$ ), mais la fertilité peut être plus faible pendant la saison d'anoestrus. Si I'IA avec de la semence congelée ou fraîche de mâles améliorés doit être utilisée, le dépôt " classique " de la semence via le cervix conduit à des taux de fertilité assez faibles, en particulier pour les taux d'ovulation élevés. Le dépôt intra-utérin de la semence sous laparoscopie conduit à des taux de fécondation équivalents à ceux obtenus après saillie naturelle (Vallet et al 1991).

Les embryons peuvent être collectés du jour 6 au jour 8 par laparotomie, qui permet des taux de récupération élevés, mais seulement une ou deux fois. La collecte sous contrôle laparoscopique doit être utilisée pour des collectes répétées sur les mêmes femelles (jusqu'à 7 collectes, Baril 1995). La collecte via le cervix n'est pas souhaitable du fait de la difficulté de pénétration dans les cornes utérines et parce que les taux de collecte restent faibles (Soonen et al 1991, Flores-Foxworth et al 1992).

La congélation des embryons caprins est possible en utilisant des techniques " classiques " dérivées de celles utilisées dans l'espèce bovine. Le développement in vitro des blastocystes est plus élevé que celui des morulae, que le cryoprotecteur soit le glycérol ou l'éthylène-glycol, alors que des morulae se développent de la même façon in vivoque des blastocystes. Pour les deux stades, le nombre d'embryons qui se développent jusqu'à terme est plus élevé lorsqu'ils sont congelés avec de l'éthylène-glycol (51 \%, $n=100)$, qu'avec du glycérol ( $30 \%, n=83$; $\mathrm{P}<0,08$, Le Gal et al 1993). La vitrification d'embryons caprins, c'est-à-dire la congélation très rapide après déshydratation, a également été réalisée avec succès (Yuswiati et Holtz 1990). Un résultat encore préliminaire indique qu'un taux de gestation identique (échographie à J 43) a été obtenu après transfert d'embryons qu'ils aient été vitrifiés ou congelés (vitrifiés : 5 gestations/7 receveuses vs congelés : $7 / 8$; Traldi et al 1997).

Le transfert doit être effectué par laparoscopie. Les résultats sont équivalents, voire meilleurs, que par laparotomie (Brebion et al 1992) et meilleurs que via le cervix (FloresFoxworth et al 1992). La nutrition des chèvres receveuses doit être adéquate avant et après transfert pour atteindre une fertilité élevée ( 25 vs $67 \%$ de mise bas chez des chèvres Angora sous-alimentées vs alimentées normalement; Mani et al 1994).

$\mathrm{Au}$ total, le nombre de chevreaux nés par femelle donneuse (collectée une seule fois) varie de 3 à 4, selon que les embryons sont congelés ou pas (figure 6 ; Baril 1995). 


\section{Production d'embryons in vitro}

Il est maintenant possible d'aboutir à un développement à terme, après transfert dans des femelles receveuses, de blastocystes complètement produits in vi tro (Crozet et al 1993, Keskintepe et al 1994). Pour produire des blastocystes, différentes étapes doivent être franchies in vitro: la maturation des ovocytes ovariens (MIV), la capacitation des spermatozoïdes et la fécondation (FIV) et les premiers stades de clivage et de développement jusqu'au stade blastocyste en culture (DIV).

Pour produire des ovocytes avec une pleine capacité de développement, il est nécessaire de les sélectionner a la fin de leur phase de croissance, lorsqu'ils acquièrent la compétence à achever la maturation méiotique et le développement embryonnaire. Les ovocytes provenant de petits follicules $(2-3 \mathrm{~mm}$ de diamètre) et de follicules moyens (3,1-5 $\mathrm{mm}$ de diamètre) conduisent à une plus faible proportion de blastocystes que ceux prélevés dans des gros follicules (plus de $5 \mathrm{~mm}$ de diamètre) : 24 vs 39 vs $53 \%$ respectivement (Cognié et al 1996). Des ovocytes ovulés in vivo, puis fécondés et cultivés in vitro dans les mêmes conditions conduisent à un taux de $70 \%$ de blastocystes (G. Baril, N. Poulin et $Y$. Cognié, résultats non publiés), ce qui indique que les conditions de maturation (in vivo ou in vi tro) peuvent également influencer l'aptitude de l'ovocyte au développement. Des progrès très importants ont éte réalisés récemment en ce qui concerne la mise au point d'un milieu optimum de maturation des ovocytes qui consiste en du fluide folliculaire caprin $(10 \%)$ et de la FSH $(100 \mathrm{ng} / \mathrm{ml})$ dans du milieu M 199 sous $5 \%$ de $\mathrm{CO}_{2}$, ce qui permet une simplification (en ne réalisant pas de co-culture avec des cellules de granulosa) en même temps qu'une meilleure efficacité de la MIV (Poulin et al 1996, Cognié et al 1996).

L'âge de la femelle donneuse peut aussi influencer la qualité de l'ovocyte. Les ovocytes collectés à partir de chèvres prépubères conduisent à une proportion plus faible de fécondations normales que ceux provenant de chèvres adultes (Martino et al 1995).

La collecte d'ovocytes à partir d'ovaires d'abattoir, par aspiration ou dissection des follicules, permet de récupérer de 1,5 à 2,1 ovocytes par ovaire (Martino et al 1994, Pawshe et al 1994). Le découpage de l'ovaire en fines lamelles a été rapporté comme permettant la récupération d'un plus grand nombre de complexes ovocytes-cumulus (6 coc/ovaire : Martino et al 1994), mais les ovocytes supplémentaires, provenant essentiellement de petits follicules, sont moins aptes à se développer après FIV (Keskintepe et al 1994). Finalement, ces trois techniques de collecte sont sensiblement équivalentes en termes d'embryons produits (Pawshe et al 1994).

En moyenne 9 coc par ovaire (incluant 4 coc provenant de follicules de taille supérieure à $5 \mathrm{~mm}$ ) peuvent être obtenus chez des chèvres prétraitées par FSH (Crozet et al 1995). Quand la collecte doit être faite sur des femelles de valeur, les ovocytes peuvent également être prélevés de façon répétée (une fois par semaine) par aspiration sous contrôle laparoscopique, ce qui permet de récupérer, après traitement FSH, 3 à 4 coc par ovaire (Todini et al 1994, Graff et al 1995). Des taux élevés de fécondation (environ $85 \%$ ) sont atteints en utilisant un milieu de culture supplémenté avec du sérum de brebis en oestrus, afin d'induire la capacitation des spermatozoïdes (De Smedt et al 1992). Ces conditions sont aussi valables pour la semence congelée (Cognié et al 1992). Après avoir écarté les oeufs polyspermiques (10 à $20 \%$ ), une moyenne de $70 \%$ d'oeufs normaux fécondés in vi tro peut être obtenue en routine. Les techniques mises au point pour la capacitation du sperme et les conditions de FIV permettent la pénétration synchrone d'ovocytes par les spermatozoïdes et, par conséquent, la production d'une population synchrone d'embryons au stade une cellule, utilisables pour I'injection de transgènes dans les pronuclei, qui doit être réalisée 14-18 heures après l'insémination. L'héparine a été rapportée comme permettant un accroissement de la pénétration des oeufs par les spermatozoïdes, lorsqu'elle est ajoutée au milieu de FIV qui contient le sérum de brebis en oestrus (Cox et al 1994), mais la qualité des embryons produits après utilisation d'héparine reste discutable (Poulin et al 1996).

Pour le DIV, la culture de jeunes embryons ( 2 à 4 cellules) en présence de cellules d'oviducte conduit à la production de significativement plus de blastocystes et de blastocystes éclos que la culture avec des cellules utérines ou dans le milieu seul (Prichard et al 1992). Désormais, avec le perfectionnement continuel des techniques de culture, un système alternatif simple, qui réduit très fortement les risques sanitaires, peut être utilisé. II s'agit d'une solution saline tamponnée (FSO : fluide synthétique d'oviducte) supplémenté avec des acides aminés et du sérum et incubé sous une atmosphère de $5 \% \mathrm{O}_{2}, 5 \% \mathrm{CO}_{2}, 90 \% \mathrm{~N}_{2}$. Dans ces conditions, l'aptitude des blastocystes à se développer jusqu'au terme après transfert est proche du taux de développement de ceux produits in vi vo $61 \%$ des blastocystes produits in vitro donnent naissance à des chevreaux; Poulin et al 1996). Enfin, des résultats encourageants sont également obtenus en ce qui concerne la survie des embryons vitrifiés après production in vi tro (A. Traldi, communication personnelle).

\section{Conclusion}

Des progrès rapides et significatifs ont été réalisés en ce qui concerne la maîtrise de la reproduction caprine, dans le domaine des différents traitements appliqués aux animaux, en ferme et dans les centres d'IA, aussi bien que dans le domaine de la manipulation in vitro des gamètes

Dans le domaine de la production de semence et de I'IA, des progrès restent à faire pour améliorer l'efficacité des techniques de

\section{La production d'embryons in vitro connaît des progrès rapides grâce à la mise au point des milieux de maturation des ovocytes et de culture des embryons.}


congélation de la semence. L'utilisation d'inhibiteurs de la lipase BUSgp60 du plasma séminal, afin d'accroître la viabilité des spermatozoïdes dans les dilueurs à base de lait et/ou de jaune d'oeuf doit être testée.

Un des principaux problèmes concernant les traitements hormonaux de maîtrise de l'oestrus et de l'ovulation est d'arriver à réduire les effets des injections répétées de eCG, qui réduisent la fertilité des femelles inséminées artificiellement. Les raisons de l'importante variabilité entre individus et la mise au point de nouveaux traitements de remplacement de eCG, sont les deux voies de recherche à développer.

Dans ce domaine de la maîtrise de l'oestrus et de l'ovulation, le développement de techniques alternatives à l'utilisation des hormones exogènes constitue également une des priorités nouvelles qui doivent faire l'objet de recherches.

Des avancées très importantes ont été réalisées en ce qui concerne la production, la col- lecte, la congélation et le transfert d'embryons in vivo, qui constituent maintenant des techniques disponibles utilisables pour l'échange d'animaux améliorés avec un risque réduit de transmission des maladies.

La production d'embryons in vitro a également connu des progres rapides et importants ces dernières années, mais les rendements des différentes étapes sont encore à améliorer afin d'aboutir à une utilisation commerciale de cette technique. On peut raisonnablement penser qu'il sera bientôt possible de produire des embryons avec le même rendement qu'in vivo, mais à un coût très inférieur.

\section{Remerciements}

Une partie des expériences présentées dans cet article ont été financées par les Régions Centre et PoitouCharentes, par le Ministère de l'Agriculture et SANOFI Santé Nutrition Animale.

\section{Références}

Aamdal J ., Lyngset O., Fossum K., 1965. Toxic effect of Iysolecithin on sperm. A preliminary report. Nordic Vet. Med., 17, 633-634.

Akinlosotu B.A., Wilder C.D 1993, Fertility and blood progesterone levels following LHRH-induced superovulation in FSH-treated anestrous goats.Theriogenology, 40, 895-904.

Baril G., 1995. Possibilidades atuais da transferência de embriões em caprinos. Proc XI Congreso Brasileiro de Reproduçao Animal, Belo Horizonte, 110-120.

Baril G., Remy B., Vallet J.C., Beckers J .F., 1992. Effect of repeated use of progestagen-PMSG treatment for estrus control in dairy goats out of breeding season. Reprod. Domestic Anim., 27, 161-168.

Baril G., Leboeuf B., Saumande J ., 1993. Synchronization of oestrus in goats: The relationship between time of occurrence of estrus and fertility following artificial insemination. Theriogenology, 40, 621-628.

Baril G., Pougnard J.L., Freitas V.J.F., Leboeuf B., Saumande J., 1996a. A new method for controlling the precise time of occurrence of the preovulatory gonadotropin surge in superovulated goats. Theriogenology, 45, 697-706.

Baril G., Remy B., Leboeuf B., Beckers J .F., Saumande J ., 1996b. Synchronization of estrus in goats: The relationship between eCG binding in plasma, time of occurrence of estrus and fertility following artificial insemination. Theriogenology, 45, 1553-1559.

Borque C., Pintado B., Perez B., Gutierrez A., Monoz I., Mateos E., 1993. Progesterone levels in superovulated Murciana goats with or without successful embryo collection. Theriogenology, 39, 192 (Abstract).

Brebion P., Baril G., Cognié Y., Vallet J .C., 1992. Transfert d'embryons chez les ovins et les caprins. Ann. Zootech. 41, 331-339.
Bretzlaff K.N., Madrid N., 1985. Synchronization of oestrus and fertility in goats with norgestomet ear implants. Theriogenology, 24, 351-357.

Chemineau P., Malpaux B., Delgadillo J.A., Guérin Y. Ravault J .P., Thimonier J., Pelletier J., 1992. Control of sheep and goat reproduction: use of light and melatonin Anim. Reprod. Sci., 30, 157-184.

Chemineau P., Malpaux B., Pelletier J., Leboeuf B., Delgadillo J.A., Deletang F., Pobel T., Brice G., 1996 Emploi des implants de mélatonine et des traitements photopériodiques pour maîtriser la reproduction saisonnière chez les ovins et les caprins. INRA Prod. Anim., 9 $45-60$

Cognié Y., Guérin Y., Poulin N., Crozet N., 1992 Successful use of frozen buck semen for in vitro fertilization of ovulated goat oocytes. Proc. 5th Intl Conf. Goats, New Delhi, India, 3, 1248-1252.

Cognié Y., Poulin N., Lamara A., 1996. The in vitro production of goat embryos using individual oocytes from different sources. Proc. 12th Association Européenne de Transfert Embryonnaire, Lyon, 122 (Abstract).

Corteel J.M., Leboeuf B., Broqua B., 1993. Identification de facteurs favorables à la fertilité des chevrettes inséminées au cours d'un oestrus induit par voie hormonale. Elevage et Insemination, 255, 1-8.

Cox J.F., Avila J., Saravia F., Santa Maria A., 1994 Assessment of fertilizing ability of goat spermatozoa by in vitro fertilization of cattle and sheep intact oocytes. Theriogenology, 41, 1621-1629.

Crozet N., De Smedt V., Ahmed-Ali M., Sevellec C., 1993. Normal development following in vitro oocyte maturation and fertilization in the goat. Theriogenology, 39, 206 (Abstract).

Crozet N., Ahmed-Ali M., Dubos M.P., 1995 Developmental competence of goat oocytes from follicles of different size categories following maturation fertilization and culture in vitro. J. Reprod. Fert., 103, 293-298. 
De Smedt V., Crozet N., Ahmed-Ali M., Martino A., Cognié Y., 1992. In vitro maturation and fertilization of goat oocytes. Theriogenology, 37, 1049-1060.

Delgadillo J .A., Leboeuf B., Chemineau P., 1991. Decrease of seasonality of sexual behaviour and sperm production in bucks by short photoperiodic cycles. Theriogenology, 36, 755-770.

Delgadillo J .A., Leboeuf B., Chemineau P., 1992. Abolition of seasonal variations in semen quality and maintenance of sperm fertilizing ability by short photoperiodic cycles in he-goats. Small Rum. Res., 9, 47-59.

Delgadillo J.A., Leboeuf B., Chemineau P., 1993. Maintenance of sperm production in bucks using a third year of short photoperiodic cycles. Reprod. Nutr. Dévelop., 33, 609-617

Delgadillo J .A., Hochereau-de Reviers M.T., Daveau A. Chemineau P., 1995. Effect of short photoperiodic cycles on male genital tract and testicular parameters in male goats (Capra hircus). Reprod. Nutr. Dévelop., 35, 549-558.

Deveson S., F orsyth I.A., Arendt J ., 1992a. Induced out-ofseason breeding in British Saanen dairy goats: use of artificial photoperiods and/or melatonin administration. Anim. Reprod. Sci., 29, 1-5.

Deveson S., Forsyth I.A., Arendt J ., 1992b. Retardation of pubertal development by prenatal long days in goat kids born in autumn. J. Reprod. Fert., 95, 629-637.

Flores-Foxworth G., McBride B.M., Kraemer D.C., Nut L.C., 1992. A comparison between laparoscopic and transcervical embryo collection and transfer in goats. Theriogenology, 37, 213 (Abstract).

Freitas V.J .F., Baril G., Saumande J ., 1996a. Induction and synchronization of oestrus in goats: the relative efficiency of one versus two fluorogestone acetate-impregnated vaginal sponges. Theriogenology, 46, 1251-1256.

Freitas V.J .F., Baril G., Bosc M., Saumande J ., 1996b. The influence of ovarian status on response to estrus synchronization treatment in dairy goats during the breeding season. Theriogenology, 45, 1561-1567.

Freitas V.J.F., Baril G., Saumande J ., 1997a. Estrus synchronization in dairy goats: use of fluorogestone acetate vaginal sponges or norgestomet ear implants. Anim. Reprod. Sci., 46, 237-244.

Freitas V.J .F., Baril G., Martin G.B., Saumande J ., 1997b. Physiological limits to further improvement in the efficiency of oestrous synchronization in goats. Reprod. Fert. Develop., 9, 551-556.

Gebbie F., 1993. Control of seasonal breeding and coat development in the goat. Ph.D. Thesis, University of Surrey, UK, $205 \mathrm{p}$.

Gootwine E., Barash I., Bor A., Dekel I., Friedler A., Heller M., Zaharoni U., Zenue A., Shani M., 1997. Factors affecting success of embryo collection and transfer in a transgenic goat program. Theriogenology, 48, 485-499.

Graff K.J., Meintjes M., Paul J.B., Dyer V.W., Denniston R.S., Ziomek C., Godke R.A., 1995. Ultrasound-guided transvaginal oocyte recovery from $\mathrm{FSH}$-treated goats for IVF. Theriogenology, 43, 223 (Abstract).

Hesselink J.W., 1993. Hydrometra in dairy goats: reproductive performance after treatment with prostaglandins. Vet. Record, 133, 186-187.

Humblot P., Brice G., Chemineau P., Broqua B., 1995. Mortalité embryonnaire chez la chèvre laitière après synchronisation des chaleurs et insémination artificielle à contre saison. Rencontres Rech. Rum., 2, 387-390.
Iritani A., Nishikawa Y., 1972. Studies on the egg yolk coagulation enzyme (phospholipase) in goat semen. IX. Enzyme concentration in the semen collected from the Cowper's gland removed goat. Memories College Agriculture Kyoto University, 101, 57-63.

Keskintepe L., Darwish G.M., Kenimer A.T., Brackett B.G., 1994. Term development of caprine embryos derived from immature oocytes in vi tro. Theriogenology, 42, 527-535.

Krisher R.L., Gwazdauskas F.C., Page R.L., Russel C.G., Caneseco R S Sparks A.E T. Velander W.H. Johnson J.L., Pearson R.E., 1994. Ovulation rate, zygote recovery and follicular populations in FSH- superovulated goats treated with PgF $2 \alpha$ and/or GnRH. Theriogenology, 41, $491-498$

Le Gal F., Baril G., Vallet J.C., Leboeuf B., 1993. In vivo and in vitro survival of goats embryos after freezing with ethylene glycol or glycerol. Theriogenology, 40, 771-

Leboeuf B., Bernelas D., Pougnard J .L., Baril G., Maurel M.C., Boué P., Terqui M., 1993. Time of ovulation after LH peak in dairy goats induced to ovulate with hormonal treatment. Proc. 9th Association Européenne de Transfert E mbryonnaire, Lyon, 226 (Abstract).

Leboeuf B., Renaud G., De Fontaubert Y., Broqua B., Chemineau P., 1994. E'chographie et pseudogestation chez la chèvre. Proc. 7th Intl Meeting Animal Reproduction, Murcia, Spain, 251-255.

Leboeuf B., Baril G., Maurel M.C., Bernelas D., Marcheteau ]. Berson Y., Broqua B., Terqui M., 1996. Effect of progestagen/PMSG repeated treatments in goats on fertility following artificial insemination (A.I.) Proc. 6th Intl Conf. Goats, Bejing, China, 2, 827.

Leboeuf B., Manfredi E., Boué P., Piacère A., Brice G., Baril G., Broqua C., Humblot P., Terqui M., 1998. L'insémination artificielle et l'amélioration génétique chez la chèvre laitière en France. INRA Prod. Anim., 11, 171-181.

Malpaux B., Chemineau P., Pelletier J., 1993. Melatonin and reproduction in sheep and goats. In : H.S. Yu and R.J. Reiter (eds), Melatonin: biosynthesis, physiological effects and clinical applications, 253-287. CRC Press, Boca Raton.

Mani A.U., Watson E.D., McKelvey W.A.C., 1994. The effects of subnutrition before or after embryo transfer on pregnancy rate and embryo survival in does. Theriogenology, 41,1673-1678.

Martino A., Mogas T., Palomo M.J ., Paramio M.T., 1995. In vitro maturation and fertilization of prepubertal goat oocytes. Theriogenology, 43, 473-485.

Martino A., Palomo M.J., Mogas T., Paramio M.T., 1994. Influence of the collection technique of prepubertal goat oocytes on in vitro maturation and fertilization. Theriogenology, 42, 859-873.

Maurel M.C., Leboeuf B., Baril G., Bernelas D., 1992. Determination of the preovulatory $\mathrm{LH}$ peak in dairy goats using an ELISA kit on farm. Proc. 8th Association Européenne de Transfert Embryonnaire, Lyon, 186 (Abstract).

Mialot J.P., Saboureau L., Gueraud J.M., Prengere E., Parizot D., Pirot G., Duquesnel R., Petat M., Chemineau P., 1991. La pseudogestation chez la chèvre : observations préliminaires. Recueil Méd. Vét., Spécial Reproduction Ruminants, 1, 383-390. 
Nowshari M.A., Beckers J.F., Holtz W., 1995. Superovulation of goats with purified pFSH supplemented with defined amounts of pLH. Theriogenology, 43, 797-802

Nunes J .F., Corteel J .M., Combarnous Y., Baril G., 1982. Rôle du plasma séminal dans la survie in vi tro des spermatozoïdes de bouc. Reprod. Nutr. Dévelop., 22, 611-620.

Pawshe C.H., Totey S.M., J ain S.K., 1994. A comparison of three methods of recovery of goat oocytes for in vitro maturation and fertilization. Theriogenology, 42, 117-125.

Pellicer-Rubio M.T., Combarnous Y., 1998. Deterioration of goat spermatozoa in skimmed milk-based extenders as a result of oleic acid released by the bulbourethral lipase BUSgp60. J . Reprod. Fert., 112, 95-105.

Pellicer-Rubio M.T., Magallon T., Combarnous Y., 1997. Deterioration of goat sperm viability in milk extenders is due to a bulbourethral 60-kilodalton glycoprotein with triglyceride lipase activity. Biol. Reprod., 57, 1023-1031.

Poulin N., Guler A., Pignon P., Cognié Y., 1996. In vitro production of goat embryos: heparin in IVF medium affects developmental ability. Proc. 6th Intl Conf. Goats, Bejing, China, 2, 838-840.

Prichard J.F., Thibodeaux J.K., Pool S.H., Blackewood E.G., Menezo Y., Godke R.A., 1992. In vitro co-culture of early stage caprine embryos with oviduct and uterine epithelial cells. Human Reprod., 7, 553-557.

Remy B., Baril G., Vallet J.C., Chouvet C., Saumande J. Chupin D., Beckers I.F., 1991. Are antibodies responsible for a decreased superovulatory response in goats wich have been treated repeatedly with porcine follicle-stimulating hormone? Theriogenology, 36, 389-399.

Roy A., 1957. Egg yolk-coagulating enzyme in the semen and Cowper's glands of the goat. Nature, 179, 318-319.

Roy F., Maurel M.C., Combarnous Y., Briois J .P., Pobel T., Deletang $F ., 1995$. Etude de la réponse immunitaire observée chez les ovins et les caprins traités avec PMSG dans le cadre de l'insémination artificielle. Rencontres Rech. Rum., 2, 395-398.
Roy F., Maurel M.C., Vaiman D., Cribiu E., Lantier I., Combarnous Y., Guillou F., 1998. Implication du complexe majeur d'histocompatibilité dans la variabilité de la réponse immunitaire humorale à la choriogonadotropine équine (eCG/PMSG) chez les caprins : approche génétique Rencontres Rech. Rum., 5, 115-117.

Soonen A.H., Lewalski S., Meinecke-Tilman S., Meinecke B., 1991. Transcervical collection of ovine and caprine embryos. Proc. 7th Sci. Meeting European Embryo Transfer Association, Cambridge, 1, 208 (Abstract).

Soulière L. 1991. La pseudogestation chez la chèvre. Aspects physiologiques et zootechniques. Mémoire ENSFA Rennes-INRA-PRMD Nouzilly, 28 p.

Todini L., Cognié Y., Poulin N., Guérin Y., 1994. Recupero in vi vo di oociti di capra mediante aspirazione follicolare per via laparoscopica. Proc. Congresso SIPAOC, 331 334.

Traldi A.S., Leboeuf B., Pougnard J .L., Baril G., Mermillod $P$ 1997. Vitrification: A cryopreservation method for embryos produced in vivo in the goat. Arq.Fac. UFRGS Porto Alegre, v. $25 n^{\circ} 1$ (abstract).

Vallet J.C., Casamitjana P., Brebion P., Perrin J., 1991. Techniques de production, de conservation et de transfert d'embryons chez les petits ruminants. Recueil Méd. Vét., Spécial Reproduction Ruminants, 167, 293-301.

Von Brackel-Bodenhausen A., Wuttke W., Holtz W., 1994. Effects of photoperiod and slow-release preparations of bromocryptine and melatonin on reproductive activity and prolactin secretion in female goats. J. Anim. Sci., 72, 955-962.

Wrathall A.E., 1995. Embryo transfer and disease transmission in livestock: a review of recent research Theriogenology, 43, 81-88.

Yuswiati E., Holtz W., 1990. Successful transfer of vitrified goat embryos. Theriogenology, 34, $629-632$.

\section{Abstract}

\section{Recent advances in goat reproduction.}

The control of goat reproduction is interesting for technical reasons ( synchronisation of kidding, adapta. tion to forage availability or to economy), and for genetic reasons (identification and dissemination of improved genotypes). The use of short-light rhythms leads to dramatically increased production of AI doses per buck and prevents the occurrence of a "resting" season. Recent identification of a bulbo-urethral lipase in goat sperm opens new perspectives in sperm preservation. Light+"short days" treatments also allow the induction of out-of-season estrous cycles and ovulations leading to enhanced fertility. Repeated use of eCG provokes the production of antibodies, delays the timing of ovulation and causes a reduction in fertility after fixed-time artificial insemination. All steps of embryo production, freezing and transfer are now controlled and allow the attainment of satisfactory rates of kids born per donor female, which are compatible with the development of the technique for exchanging genotypes between countries. In vitro production of embryos allows high development rates to be achieved after in vitro maturation and fertilisation of oocytes, and will ensure the production of synchronous populations of one-cell zygotes at the stage required by new biotechnologies.

Chemineau P., Baril G., Leboeuf B., Maurel M.C., Roy F., Pellicer-Rubio M., Malpaux B., Cognié $Y$., 1999. Implications des progrès récents en physiologie de la reproduction pour la conduite de la reproduction dans l'espèce caprine. INRA Prod. Anim., 12, 135-146. 